\title{
Rapid, semi-automatic fracture and contact mapping for point clouds, images and geophysical data
}

\author{
Samuel T. Thiele, Lachlan Grose, Anindita Samsu, Steven Micklethwaite, Stefan A. Vollgger, and \\ Alexander R. Cruden
}

School of Earth, Atmosphere and Environment, Monash University, Melbourne, 3800, Australia

Correspondence: Samuel T. Thiele (sam.thiele@monash.edu)

Received: 3 August 2017 - Discussion started: 15 August 2017

Revised: 14 November 2017 - Accepted: 16 November 2017 - Published: 21 December 2017

\begin{abstract}
The advent of large digital datasets from unmanned aerial vehicle (UAV) and satellite platforms now challenges our ability to extract information across multiple scales in a timely manner, often meaning that the full value of the data is not realised. Here we adapt a least-cost-path solver and specially tailored cost functions to rapidly interpolate structural features between manually defined control points in point cloud and raster datasets. We implement the method in the geographic information system QGIS and the point cloud and mesh processing software CloudCompare. Using these implementations, the method can be applied to a variety of three-dimensional (3-D) and two-dimensional (2-D) datasets, including high-resolution aerial imagery, digital outcrop models, digital elevation models (DEMs) and geophysical grids.

We demonstrate the algorithm with four diverse applications in which we extract (1) joint and contact patterns in high-resolution orthophotographs, (2) fracture patterns in a dense 3-D point cloud, (3) earthquake surface ruptures of the Greendale Fault associated with the $M_{\mathrm{w}} 7.1$ Darfield earthquake (New Zealand) from high-resolution light detection and ranging (lidar) data, and (4) oceanic fracture zones from bathymetric data of the North Atlantic. The approach improves the consistency of the interpretation process while retaining expert guidance and achieves significant improvements $(35-65 \%)$ in digitisation time compared to traditional methods. Furthermore, it opens up new possibilities for data synthesis and can quantify the agreement between datasets and an interpretation.
\end{abstract}

\section{Introduction}

Remote sensing datasets are commonly used in the earth sciences to interpret the morphology, location, timing and orientation of geological features. These data types, now routinely delivered by satellite, aerial and UAV platforms, have advanced to the point at which they are widely available at high resolution and in some instances are frequently updated. This proliferation of data has led to a situation in which it is now no longer practical to use manual methods to extract geological information, meaning that the full geological value of high-quality datasets is often not extracted. For example, high- and ultra-high-resolution (centimetre to millimetre) photorealistic reconstructions of geological outcrops ("digital outcrop models") are becoming widely available (McCaffrey et al., 2005; Pringle et al., 2006; Vollgger and Cruden, 2016), typically acquired using either laser scanning technology (Buckley et al., 2008) or photogrammetric workflows (Bemis et al., 2014; Smith et al., 2015). These techniques, combined with inexpensive and easy-to-use UAV technology, now make it feasible to acquire topographic data at millimetre to centimetre resolution over areas of several square kilometres (e.g. Vollgger and Cruden, 2016; Cruden et al., 2016), providing for the first time an objective method for rapidly collecting detailed 3-D information on geological structures.

There has recently been significant effort to develop automatic or computer-assisted methods for digitising structural data, in particular from orthorectified photographs or image sequences (Seers and Hodgetts, 2016; Vasuki et al., 2014; Jones et al., 2009). Achieving satisfactory automated digitisation is challenging for the mapping of geological structures due to intrinsic variables such as geometry, soft linkage 
and segmentation over multiple scales and extrinsic variables such as natural variations in colour, shadows, glare and/or incomplete geological exposure. Due to this complexity, fully automatic methods often require significant manual adjustment and vetting to remove false positives while retaining real geological features (Vasuki et al., 2014; Seers and Hodgetts, 2016).

In this paper, we first review existing approaches to the mapping of geological structures from digital data and then describe a novel least-cost-path method that can "follow" structure traces between user-defined control points in both 2-D gridded datasets (imagery, geophysical rasters, etc.) and dense 3-D point clouds (digital outcrop models). We then describe its implementation in two widely used software packages (QGIS and CloudCompare) and introduce four applications demonstrating the efficacy of the method for mapping outcrop structures, earthquake surface ruptures and oceanic fracture zones.

\section{Existing methods}

Many automated methods have been developed to extract linear features in the geosciences (e.g. Jinfei and Howarth, 1990; Tzong-Dar and Lee, 2007; Holden et al., 2012; Masoud and Koike, 2017). These use computer vision algorithms for edge and lineament detection and, while often successful in ideal situations, require substantial fine tuning to achieve optimal performance on real-world data. They also have a tendency to detect many false positives related to non-geological features such as shadows, roads or vegetation (Vasuki et al., 2014). Hence, even fully automated methods currently require significant manual effort to remove nongeological features while ensuring that features of interest are correctly detected.

To circumvent these difficulties, several methods have been developed which remain user driven but also use computational power to optimise the interpretation process and improve objectivity and consistency. Vasuki et al. (2014), for example, use an edge detection algorithm (phase congruency; Kovesi, 1999) on orthophotographs to optimise manually defined fracture traces and contacts. This allows the user to quickly define the approximate locations of interesting features and then automatically refine them, speeding up the digitisation process significantly while avoiding problems associated with false positives. Similar computer-assisted approaches have also been applied to improve the interpretation of faults in regional magnetic surveys (Holden et al., 2016) and oceanic fracture zones in global gravity datasets (Wessel et al., 2015).

In many situations, the 3-D orientations of detected features are of interest. This is typically calculated using a digital elevation model to add height information to each trace and then computing a best-fit plane (e.g. Dering et al., 2016; Jaboyedoff et al., 2009; Banerjee and Mitra, 2005). While this method works well in topography containing slopes $<45^{\circ}$, it is inherently limited to 2.5 dimensions $(2.5-\mathrm{D})$ as elevation data is gridded in the $\mathrm{X}-\mathrm{Y}$ plane, causing problems when features cross-cut steep or overhanging topography (Pavlis and Mason, 2017). For this reason, direct analysis of 3-D point cloud data is preferable over methods that are limited to 2.5-D. Unfortunately, the unstructured nature of 3-D point data means that methods for trace detection in raster data, including those previously described, cannot be easily applied.

A number of automatic methods for analysing point cloud data have been proposed. These use clustering or planefitting algorithms to automatically segment and extract fracture or bedding faces (facets) exposed on the surface of the outcrop, with reasonable success (e.g. Dewez et al., 2016; Lato and Vöge, 2012; García-Sellés et al., 2011). However, structural surfaces are not always directly exposed and instead intersect the outcrop surface to form linear features referred to as structural traces. These traces cannot be detected using facet-based techniques and require a different approach.

Seers and Hodgetts (2016) demonstrate one such approach and automatically extract 3-D structural traces by applying image-based edge detection techniques (phase congruency) to a set of images and then projecting the identified traces into 3-D using depth information derived from photogrammetric reconstructions or associated laser scan data. This approach uses multiple images to overcome issues associated with out-of-plane geometry; however, as with other fully automated methods, a variety of parameters and thresholds require careful calibration and the results must be manually vetted to remove false positives.

\section{Method: a least-cost-path approach to digital mapping}

\subsection{Theory}

The approach presented here couples algorithms for solving least-cost-path problems with both general and case-specific cost functions to capture structural features in both point cloud and raster datasets. Least-cost-path algorithms have previously been used to detect linear features in a variety of image data and have proven robust even when signal-to-noise ratios are very low (Sun and Pallottino, 2003; Vincent, 1998; Buckley and Yang, 1997).

Conceptually the algorithm can be divided into two steps, although for performance reasons our implementation performs these simultaneously. In the first step, data points (points in a point cloud or pixels in an image; Fig. 1a) are linked with their nearest neighbours using a spherical search radius slightly larger than the dataset resolution to produce a neighbourhood network (Fig. 1b). The costs of moving along links in this network (hereafter referred to as "edges") are 
(a) Input data

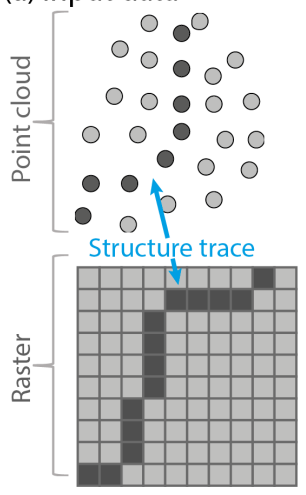

(b) Assign edges and costs

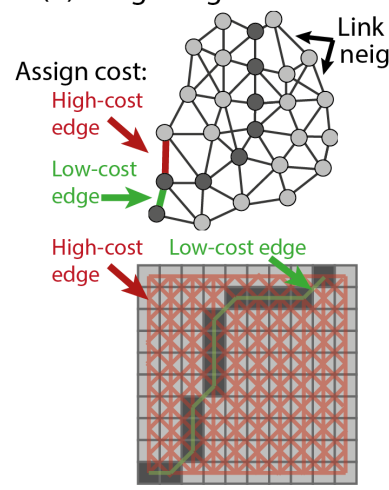

(c) Solve least-cost path

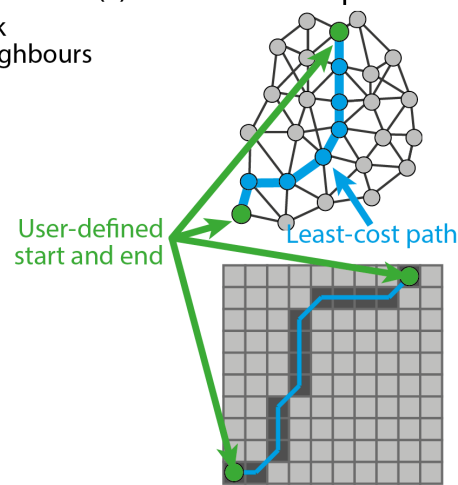

Figure 1. Schematic representation of the least-cost-path approach to trace detection for point cloud (top) and raster (bottom) data. Points and pixels on the structural trace have a lower brightness in this example (a), so a brightness-based cost function will result in low-cost edges between adjacent points and pixels that both fall on the structure trace (b). A least-cost-path calculation (c) then provides an estimate of the structure trace.

then calculated using a cost function designed to promote movement along structural or lithological traces and inhibit movement across them (Fig. 1c).

In the second step, an optimised version of Dijkstra's algorithm (Dijkstra, 1959) is used to derive the least-cost path between user-defined control points, providing the estimated trace (Fig. 1c). Dijkstra's algorithm, in essence, progressively "grows" least-cost paths from the start point until the end is found. We optimise this by requiring paths to move closer to the end at each step, thereby eliminating tortuous geometries that tend not to be geologically feasible. Once a trace has been estimated, manual adjustments can be easily applied by adding intermediate waypoints and recalculating the relevant least-cost paths.

The critical component in this approach is the cost function. A well-designed cost function produces low values for edges following structure or contact traces and high values for edges outside or cross-cutting traces. Our optimised implementation of Dijkstra's algorithm then follows edges with the lowest cost values in order to map out the feature of interest. Conveniently, simple cost functions such as point or pixel brightness or local colour gradient work well on most geological datasets; the examples presented below all map a single scalar attribute in the dataset directly to cost (point or pixel brightness for study 1 and 2, topographic slope for study 3 and bathymetric depth and vertical gravity gradient for study 4). We have designed and implemented these and several other simple cost functions that give reasonable results for different structure and data types. Specific equations for these are included in Appendix A.

\subsection{Implementation}

The above methodology has been implemented as plugins for Cloud Compare (Girardeau-Montaut, 2015) and QGIS (QGIS, 2011), both of which are cross-platform, open-source and widely used software packages for geospatial analysis. Our CloudCompare plugin (Compass) is written in $\mathrm{C}++$ and works for point cloud data, while the QGIS version (GeoTrace) is implemented as a Python plugin using numpy (van der Walt et al., 2011) and scikit-image (van der Walt, 2014) to apply our method to raster data.

Compass is bundled with the default CloudCompare distribution (since version 2.9), and the source code is freely available at https://github.com/CloudCompare/CloudCompare. Similarly, GeoTrace can be found on the QGIS plugin repository (https://plugins.qgis.org/plugins/), and the source code can be downloaded from https://github.com/lachlangrose/ GeoTrace. Complete documentation for the plugins is found at the CloudCompare wiki (http://www.cloudcompare.org/ doc/wiki/) and on the GeoTrace GitHub page.

In addition to our method for rapidly extracting structural traces, a variety of other functionality has been implemented, including tools for measuring surface orientations, lineations and true thicknesses in the Cloud Compare plugin as well as DEM-based plane-fitting and orientation analysis in the QGIS plugin.

\section{Case studies}

To demonstrate the capability of our computer-assisted trace detection approach, we present the results of four case studies. These studies highlight the versatility of our method and its increased efficiency compared to established manual methods.

The first case study involves the interpretation of joint sets in two $10 \times 10 \mathrm{~m}$ areas from a $\sim 1 \mathrm{~cm}$ resolution orthophotograph of a wave-cut platform at Bingie Bingie Point, New South Wales, Australia. The outcrop contains several Cretaceous to Paleogene dykes intruding Devonian diorites and tonalities cross-cut by a series of complex joint sets. 


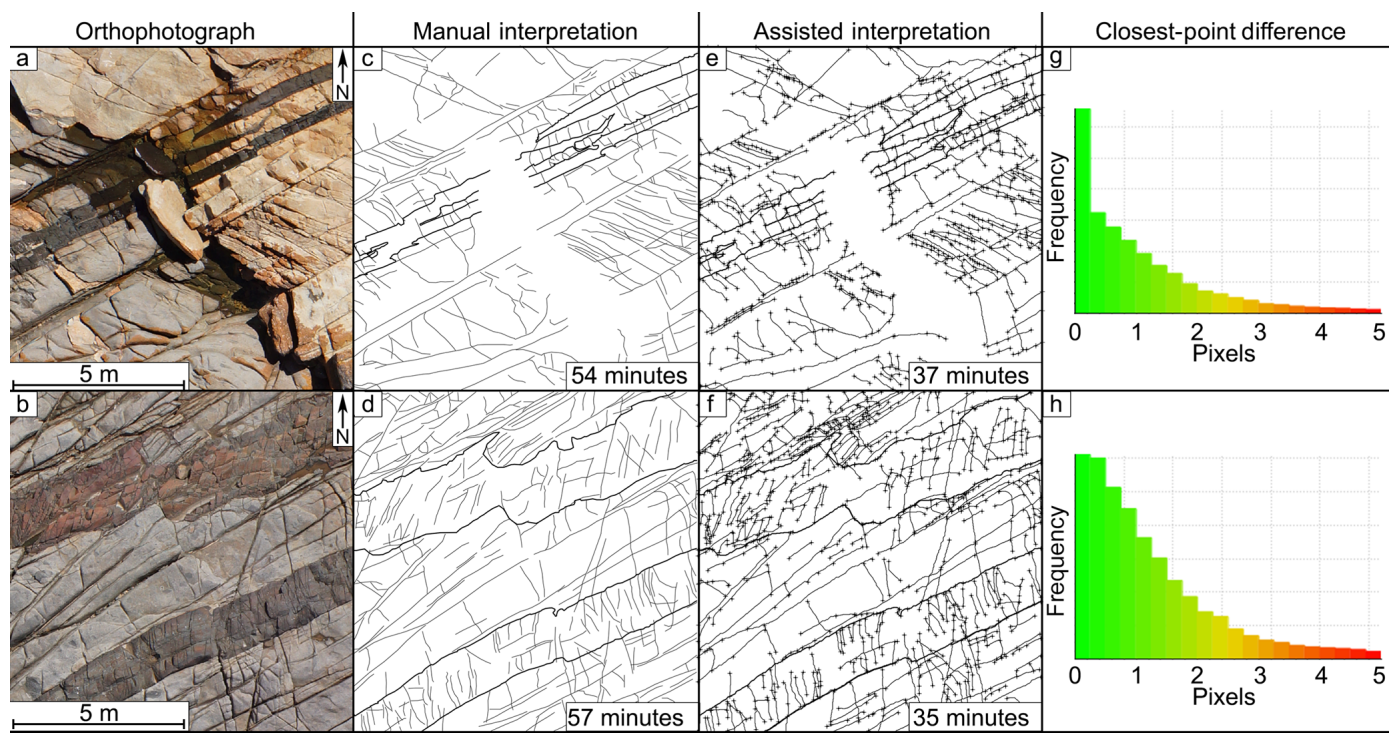

Figure 2. The two $10 \times 10 \mathrm{~m}$ orthophotographs $(\mathbf{a}, \mathbf{b})$ interpreted in study 1 . Fracture traces were digitised manually (c, d) and with our assisted method (e, f). Closest-point distances between the assisted and manual interpretations are also shown (g, h). Note that the tails of these distributions have been clipped to $5 \mathrm{~cm}$, as some assisted traces did not have manual equivalents and hence gave incorrectly large closest-point differences. Small crosses in (e) and (f) represent the control points that were digitised by the user to constrain the shortest-path algorithm.

The orthophotograph was generated by applying a structurefrom-motion multi-view stereo (SfM-MVS) workflow, as implemented in Agisoft Photoscan v1.2.6, to 297 digital photographs captured from a DJI S800 EVO multi-rotor UAV fitted with a 24.3 megapixel Sony Nex-7 camera and a $16 \mathrm{~mm}$ F2.8 prime lens (Cruden et al., 2016). The two $10 \times 10 \mathrm{~m}$ areas (Fig. 2a, b) were selected from the survey as they contain well-exposed dykes and joint sets as well as common confounding effects such as shadows and puddles. For demonstration purposes the selected areas are relatively small, but the workflow is equally applicable to much larger outcrops.

Our second case study focuses on the extraction of 3-D joint traces and orientations, which are interpreted directly on a dense 3-D point cloud. The Cape Woolamai sea stacks, located approximately $115 \mathrm{~km}$ southeast of Melbourne (Australia) on Phillip Island, have formed through the erosion of the coarse- to medium-grained Cape Woolamai granite, which intruded Silurian to lower Devonian meta-turbidites during or slightly after the mid-Devonian Tabberabberan Orogeny (a widespread episode of deformation and plutonism across Victoria; Gray, 1997; Richards and Singleton, 1981). Several generations of systematic and non-systematic joints cross-cut this granite, likely related to the cooling of the intrusion and the subsequent deformation and unloading.

For this study, a DJI Inspire 1 multi-rotor UAV and a $20 \mathrm{~mm}$ fixed focal Zenmuse X3 camera were used to capture 274 aerial photographs, which were subsequently processed using Agisoft Photoscan v1.2.6. A $45 \times 40 \times 25 \mathrm{~m}$ section of the resulting model containing a single sea stack was then extracted, containing $\sim 2$ million points and representing an average ground sampling distance of $\sim 2.5 \mathrm{~cm} \mathrm{pixel}^{-1}$. The topographic complexity of this sea stack allows for accurate joint-orientation measurement, but makes interpretation from 2.5-D datasets (orthophotograph + DEM) impractical (Fig. 3a, b).

For the third case study, surface ruptures that formed along the Greendale Fault after the $2010 M_{\mathrm{w}} 7.1$ Darfield earthquake are extracted from a $1 \mathrm{~m}$ resolution lidar-derived DEM. The data were collected a few days after the earthquake and were used along with a variety of other data to measure the surface displacement resulting from the earthquake and to interpret the kinematics of the Greendale Fault (Duffy et al., 2012).

Finally, for our last case study we interpret oceanic fracture zones in the North Atlantic from 30 arcsec bathymetry (Weatherall et al., 2015) and vertical gravity gradient data (Sandwell et al., 2014). From their inception at mid-ocean ridges, fracture zones can be used to constrain plate motion vectors and are widely used in tectonic reconstruction (Williams et al., 2016; Sandwell et al., 2014). Both these datasets provide an opportunity to test our method on globalscale geophysical data.

For each of these case studies, we also assess the similarity of our assisted results to manually derived interpretations. The aim of these comparisons is not to rigorously validate the computer-assisted method because (1) given enough control points our method could match any interpretation and (2) manual interpretation of structures from remotely sensed data is notoriously subjective, so differing results can be expected from different operators and scales (Bond et al., 2007; 

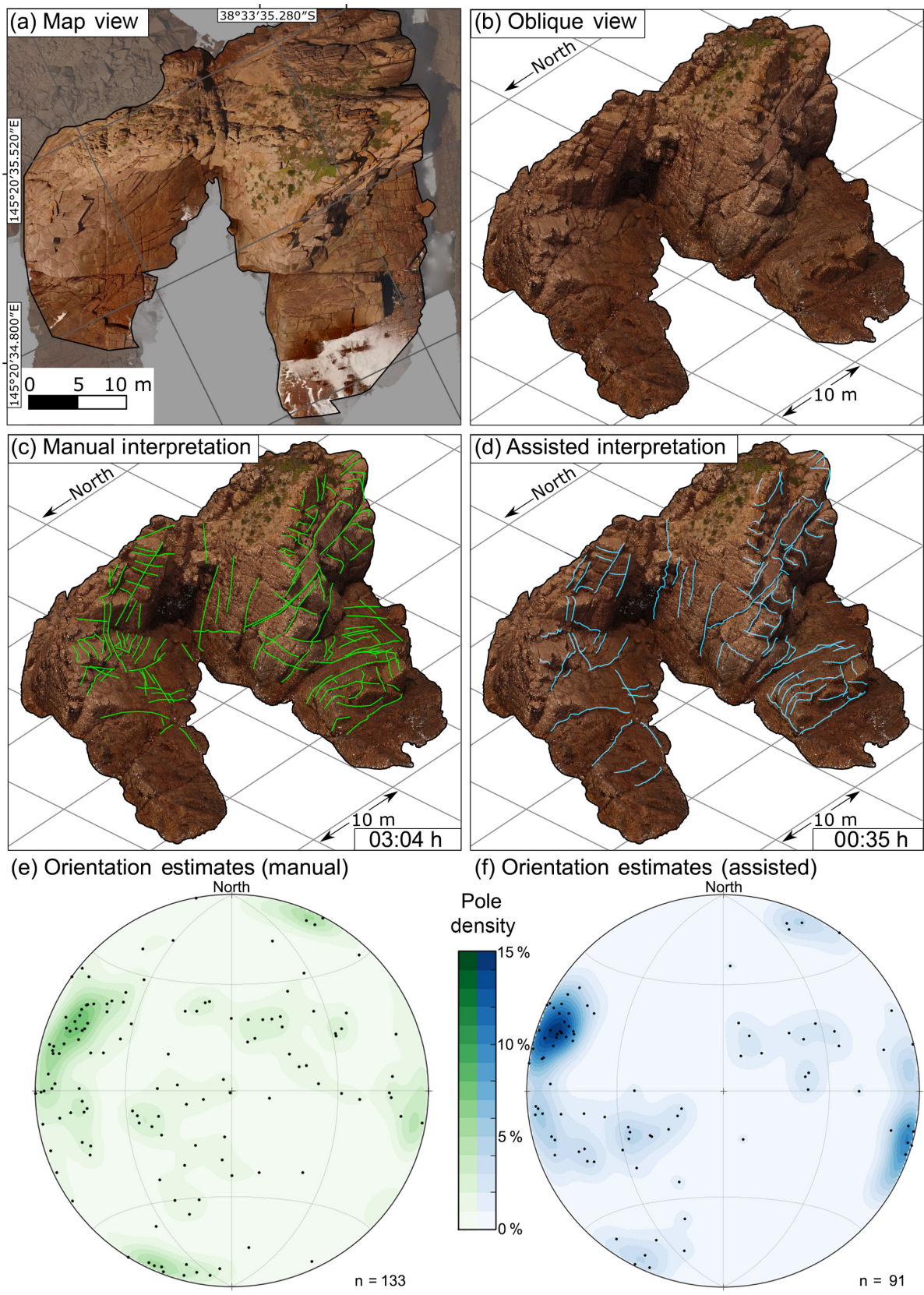

Figure 3. Orthophotograph of the Cape Woolamai sea stacks (a) and oblique view of the equivalent dense point cloud (b). A 2.5-D analysis conducted using the orthophotograph would significantly under-sample the moderately to shallowly dipping joint sets which are clearly visible on sub-vertical exposures in (b). Hence fractures were digitised in 3-D both manually (c) and using the computer-assisted approach (d). Equal-area lower hemisphere stereographic projections of poles to joint orientations estimated from each of these interpretations (e-f) show that both methods produce similar results. Poles from the computer-assisted dataset cluster more tightly (maximum density $=14.7 \%)$ than the manually interpreted dataset (maximum density $=8.1 \%$ ), indicating that the computer-assisted approach results in more consistent orientation estimates.

Scheiber et al., 2015). Instead, we seek to demonstrate the applicability and versatility of the assisted approach and to show that similar results to a manual interpretation can be produced for less time and effort.

For each interpretation, the operator was instructed to digitise every structural feature within the dataset. To ensure that this was an achievable task, the extent of the dataset used in each case study is small compared to its resolution. No attempt was made to ensure that the same number of features was extracted from each dataset, as this would affect timing measurements. Digitisation was performed at or close to the dataset resolution so that the manual interpretations con- 
Table 1. Manual vs. computer-assisted digitisation for the different study areas. Percentage improvements are calculated by comparing the average time and mouse clicks per digitised trace. Each case study shows a clear reduction in digitisation time, especially for the 3-D datasets for which manual interpretation can be especially tedious.

\begin{tabular}{|c|c|c|c|c|c|}
\hline Method & $\begin{array}{l}\text { Digitisation } \\
\text { time (h:min) }\end{array}$ & $\begin{array}{r}\text { Number of } \\
\text { traces }\end{array}$ & $\begin{array}{r}\text { Improvement } \\
\%\end{array}$ & $\begin{array}{l}\text { Number of } \\
\text { mouse clicks }\end{array}$ & $\begin{array}{r}\text { Improvement } \\
\%\end{array}$ \\
\hline \multicolumn{6}{|c|}{ Study 1: Bingie Bingie } \\
\hline \multicolumn{6}{|l|}{ Area 1: } \\
\hline Manual & $0: 54$ & 270 & - & 2253 & - \\
\hline Assisted & $0: 37$ & 283 & $35 \%$ & 917 & $61 \%$ \\
\hline \multicolumn{6}{|l|}{ Area 2: } \\
\hline $\begin{array}{l}\text { Manual } \\
\text { Assisted }\end{array}$ & $\begin{array}{l}0: 57 \\
0: 35\end{array}$ & $\begin{array}{l}338 \\
383\end{array}$ & $46 \%$ & $\begin{array}{l}2509 \\
1122\end{array}$ & $61 \%$ \\
\hline \multicolumn{6}{|c|}{ Study 2: Cape Woolamai } \\
\hline Manual & $3: 04$ & 146 & - & 6026 & - \\
\hline Assisted & $0: 56$ & 114 & $61 \%$ & 1703 & $64 \%$ \\
\hline \multicolumn{6}{|c|}{ Study 3: Greendale Fault } \\
\hline Manual & $0: 18$ & 74 & - & 1039 & - \\
\hline Assisted & 0:07 & 93 & $51 \%$ & 282 & $66 \%$ \\
\hline \multicolumn{6}{|c|}{ Study 4: Oceanic fracture zones } \\
\hline Manual & $1: 17$ & 432 & - & 5731 & - \\
\hline Assisted & $0: 35$ & 310 & $36 \%$ & 1265 & $69 \%$ \\
\hline
\end{tabular}

tained similar detail to the assisted approach (which always follows traces at the resolution of the dataset), although the operator was able to freely zoom in and out to inspect the data over multiple scales. The same operator performed the manual and assisted interpretations for case studies 1 and 2, while different operators generated each of the assisted and manual interpretations for case studies 3 and 4 . Manual interpretation in QGIS (case studies 1, 3 and 4) was performed by digitising polyline features to a shapefile using the "Add feature" tool, while in CloudCompare (case study 2) the "Trace polyline by point picking" tool was used.

\section{Results}

The time required to extract comparable amounts of structural data was significantly reduced using the computerassisted method (Table 1). This efficiency increase was especially pronounced $(61 \%)$ for the point cloud example, as manual methods for digitising linear features on 3-D point clouds are particularly time consuming.

The following four subsections compare and contrast the results of both manual and assisted interpretations in more detail.

\subsection{Bingie Bingie Point}

Both areas (Fig. 2a, b) of the Bingie Bingie Point orthophotographs contain joints over a range of scales and in a variety of host rocks, as well as features that make automated interpretation challenging such as water, shadows and debrisfilled joints. Fracture and contact traces were digitised manually in QGIS (Fig. 2c, d) and with the GeoTrace implementation of our assisted method (Fig. 2e, f). For the assisted interpretation, different cost functions were used to pick the fractures and the dyke contacts. Fractures in the orthophotographs are clearly darker than their surroundings, so a greyscale version of the orthophotograph (easily calculated using GeoTrace) was used to define the shortest-path cost function during fracture digitisation. Dyke contacts were mapped using a cost function derived from the inverse of the local brightness gradient (high gradient means low cost). This was achieved by applying a Sobel filter (essentially a local gradient operator; Sobel, 1990) to the greyscale image using scikit-image functionality (van der Walt et al., 2014) integrated into GeoTrace.

The results are visually similar to the manually derived reference interpretation (Fig. 2c-f). Closest-point differences, calculated by subsampling closely spaced points from each assisted trace and computing the shortest distance between these and a manually interpreted trace, show that the majority of traces $(78 \%$ in area 1 and $70 \%$ in area 2 ) match to 


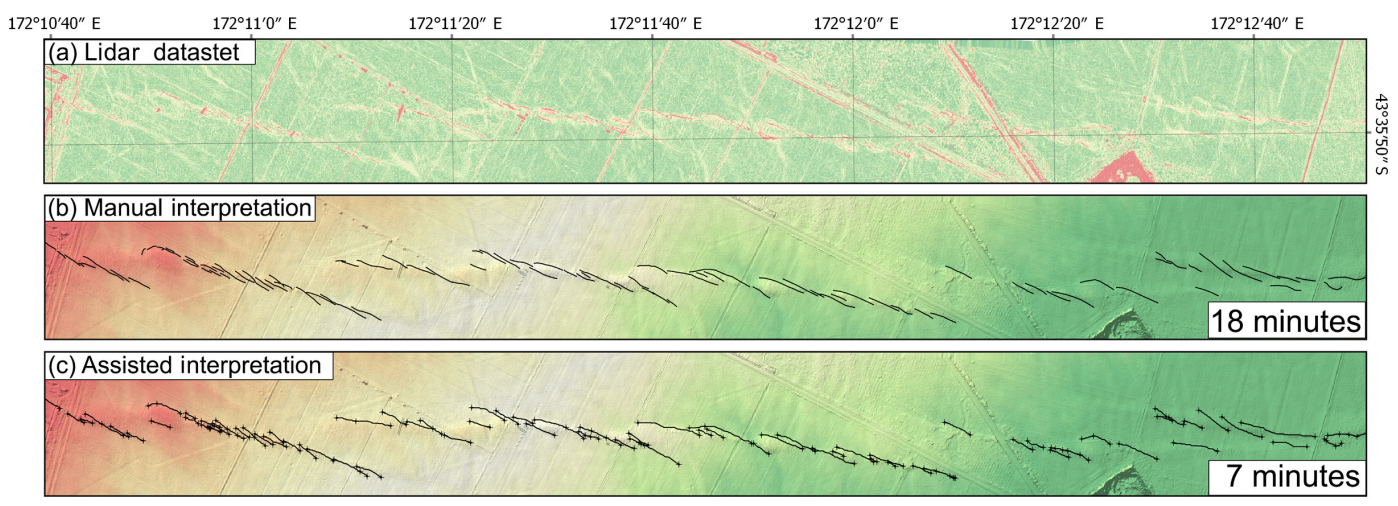

(d) Closest-point difference
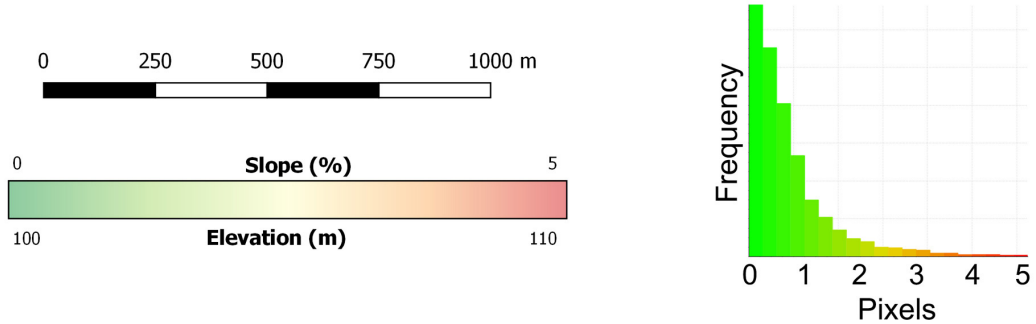

Figure 4. Slope map derived from the Greendale lidar dataset showing surface ruptures of a section of the Greendale Fault, New Zealand collected shortly after the $M_{\mathrm{W}} 7.1$ Darfield earthquake (a). Traces interpreted manually (b) and using the GeoTrace implementation of our least-cost-path method (c) are essentially equivalent (d). Control points for the assisted interpretation are shown as small crosses. Background for (b) and (c) shows the elevation.

within 2 pixels $(\approx 2 \mathrm{~cm})$, which is smaller than the ambiguity of the dataset.

\subsection{Cape Woolamai}

Joints in the Cape Woolamai digital outcrop model were interpreted in 3-D using CloudCompare, first with the manual "draw polyline" tool and then using the Compass implementation of our method. The complex topography of the sea stacks makes 2.5-D analysis inappropriate (Fig. 3a, b). As in the Bingie Bingie example, cost was defined by point brightness as fractures are defined by their darker colour.

In total, 146 joint traces were interpreted manually over $\sim 3 \mathrm{~h}$, while 114 joint traces were digitised using the Compass plugin in less than $1 \mathrm{~h}$ (Table 1). Joint orientations were estimated by calculating the least-squares plane-of-best-fit for each trace. The ratio between the second and third eigenvectors of each trace was then used to reject arbitrary planes resulting from sublinear traces using a planarity threshold of 0.75 (see Thiele et al., 2015, for a more detailed description of this method). Compass does this in real time during the digitisation processes, while orientation estimates from the manually digitised dataset were calculated after processing. The manual and computer-assisted methods resulted in 133 and 91 orientation estimates respectively.

Both sets of interpreted traces and associated orientation estimates appear to be broadly consistent for each method (Fig. 3c-f). Significantly, orientation estimates from the computer-assisted method form more pronounced clusters than equivalents estimated using the manually digitised traces. Although far from conclusive, this indicates that the computer-assisted approach improves the consistency and precision of the orientation estimates, likely due to the larger number of points it samples. The assisted method samples every point along each trace, while the manual method only includes the polyline vertices created during digitisation, making the best-fit plane more susceptible to errors caused by outliers.

\subsection{Greendale Fault}

Surface ruptures of the Greendale Fault form a series of en échelon fault scarps visible in the lidar dataset (Fig. 4a). Our shortest-path method can be used to pick the fault scarps using a cost function in which slope maps inversely with cost. This was achieved by calculating a slope raster using the QGIS DEM (Terrain Models) tool and inverting it using GeoTrace.

As in the previous examples, the assisted interpretation achieved very similar results to a manual interpretation in about half the time. Closest-point-difference calculations between the manual and assisted traces show that the two sets of interpretations are consistently within $\sim 1-2$ pixels $(\sim 2 \mathrm{~m})$. 

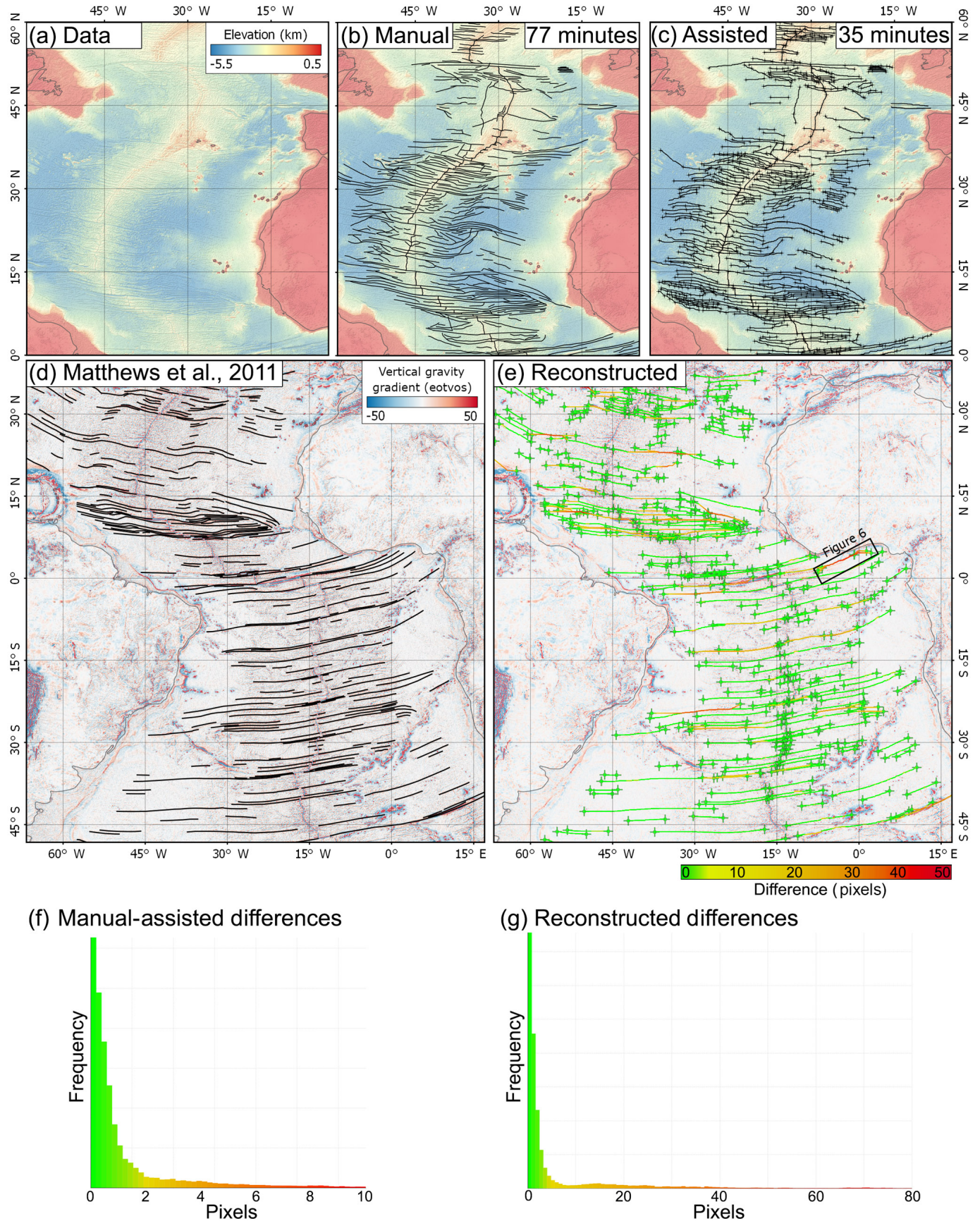

Figure 5. Bathymetry showing oceanic fracture zones in the North Atlantic (a) and associated manual (b) and assisted (c) interpretations. Fracture zones interpreted manually (d) from vertical gravity gradient by Matthews et al. (2011) and reconstructed in GeoTrace using the start and end points only (e) are also shown. As in the previous case studies, most equivalent manual and assisted traces fall within 2 pixels (f), though differences of up to 80 pixels occur in the reconstructed dataset (g). The location of Fig. 6 is shown in (e) for reference.

\subsection{Oceanic fracture zones}

Oceanic fracture zones in the North Atlantic were digitised in GeoTrace using bathymetric depth to define trace cost. Comparison with an interpretation that was digitised manually shows similar accuracies to the previous case studies, with the majority of traces within $\sim 2$ pixels and an improvement of $36 \%$ in per-trace digitisation time (Fig. 5).

Additionally, we used the start and end points of oceanic fracture zones interpreted by Matthews et al. (2011), which are based on a 2009 gravity gradient compilation (Sandwell and Smith, 2009), to constrain an otherwise unguided Geo- 

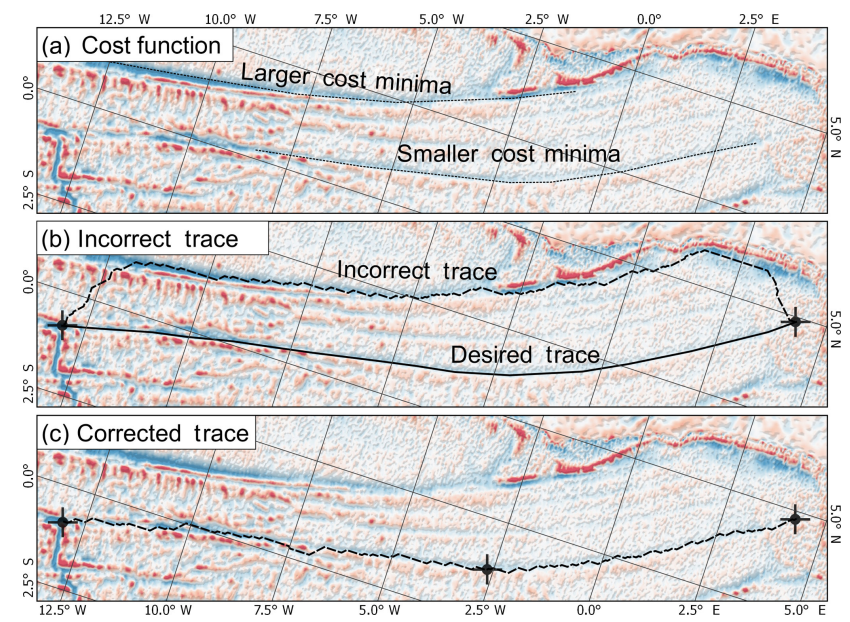

Figure 6. Example of larger cost minima (a) causing the incorrect reconstruction (b) of an oceanic fracture zone. In this case the trace can be corrected by adding a single additional control point midway along the fracture zone (c).

Trace interpretation of an updated vertical gravity gradient dataset (Sandwell et al., 2014). This was achieved using the vertical gravity gradient directly as the cost function, such that traces follow areas of low vertical gradient, and then solving the shortest path between the Matthews et al. (2011) start and end points.

The results (Fig. 5e) again highlight the tool's general accuracy, with $65 \%$ of traces falling within 2 pixels of the Matthews et al. (2011) interpretation and $79 \%$ within 5 pixels. Most errors occurred in areas of closely spaced fracture zones where the computed shortest path for many fracture zones would "detour" through adjacent low-cost features (Fig. 6). A small number of additional control points along these traces would resolve this issue (Fig. 6c) by forcing the computed path to stay in the local cost minima (the desired fracture zone) rather than taking advantage of larger adjacent minima.

\section{Discussion}

The four case studies presented above highlight applications of the least-cost-path method to the interpretation of highresolution aerial orthophotographs, 3-D point clouds, lidar DEMs and bathymetric data - all datasets commonly used in the earth sciences to interpret and characterise geological features. We discuss here three aspects of our least-cost-path approach: it improves objectivity and reproducibility, allows for automatic refinement if better data become available and, unlike fully automated methods, works in cooperation with expert guidance.

Firstly, the approach is more objective than manual digitisation. Although not as objective as fully automated methods (the location of the trace start and end are interpreted), most of the length of each trace is determined algorithmically and hence will consistently locate in the same spot. Indeed, as demonstrated in Fig. 7, the calculated shortest path varies only slightly when control points are interpreted at different locations. The results from study 2 indicate that this improved consistency might increase the precision of the derived orientation estimates (Fig. 3e, f). Furthermore, each control point can easily be stored, providing a record of the locations at which interpretive decisions were made.

Secondly, similarly to the method outlined by Wessel et al. (2015) for extracting oceanic fracture zones, these control points can also be reused to generate an updated interpretation if higher-resolution or more accurate information becomes available. This possibility is demonstrated in study 4 , in which published oceanic fracture zones were reconstructed automatically using an updated underlying dataset and the start and end points of a previous interpretation (Matthews et al., 2011). Although some quality control is required after such an operation, the digitisation process no longer needs to be completely repeated, and interpretations can be rapidly updated as datasets evolve.

When multiple datasets are available, the similarity and total cost of paths reconstructed using different datasets can be used to quantitatively assess the degree to which different datasets support an interpretation. It is common in the geosciences to bring interpretations from multiple types of data into a single synthesis (e.g. Seton et al., 2016; Blaikie et al., 2017), especially when using geophysical datasets such as gravity and magnetics. Limiting factors during such data synthesis include both the time required and the highly subjective nature of multi-data-type interpretations, so a method for rapidly quantifying the extent to which different datasets support an interpretation serves as an important addition. Similarly, sensitivity analyses could be performed by randomly moving control points and measuring the response of the traces to quantify the robustness of the interpretation to uncertainty (similar to Fig. 7).

The time it takes for users to interpret datasets using GeoTrace or Compass will vary significantly between users, and the purpose of this study was not to comprehensively measure the efficiency of our approach. Nevertheless, in each of the case studies, our initial assessment indicates that computer-assisted interpretation required $\sim 35-66 \%$ less user effort, as measured by both average time and mouse clicks per structure trace, when compared to manual methods (Table 1). The resulting traces also appear to be comparable to manual traces in each case ( \pm 2 pixels), demonstrating that our method can be used to achieve equivalent results.

The Compass implementation of the technique produces especially impressive results, reducing interpretation time in the Cape Woolamai example by $61 \%$. This is pertinent given the rapid growth in both size and availability of highresolution point cloud data and the limited range of available tools for extracting structural data from them. Significantly, the implementation of our least-cost-path method in Com- 


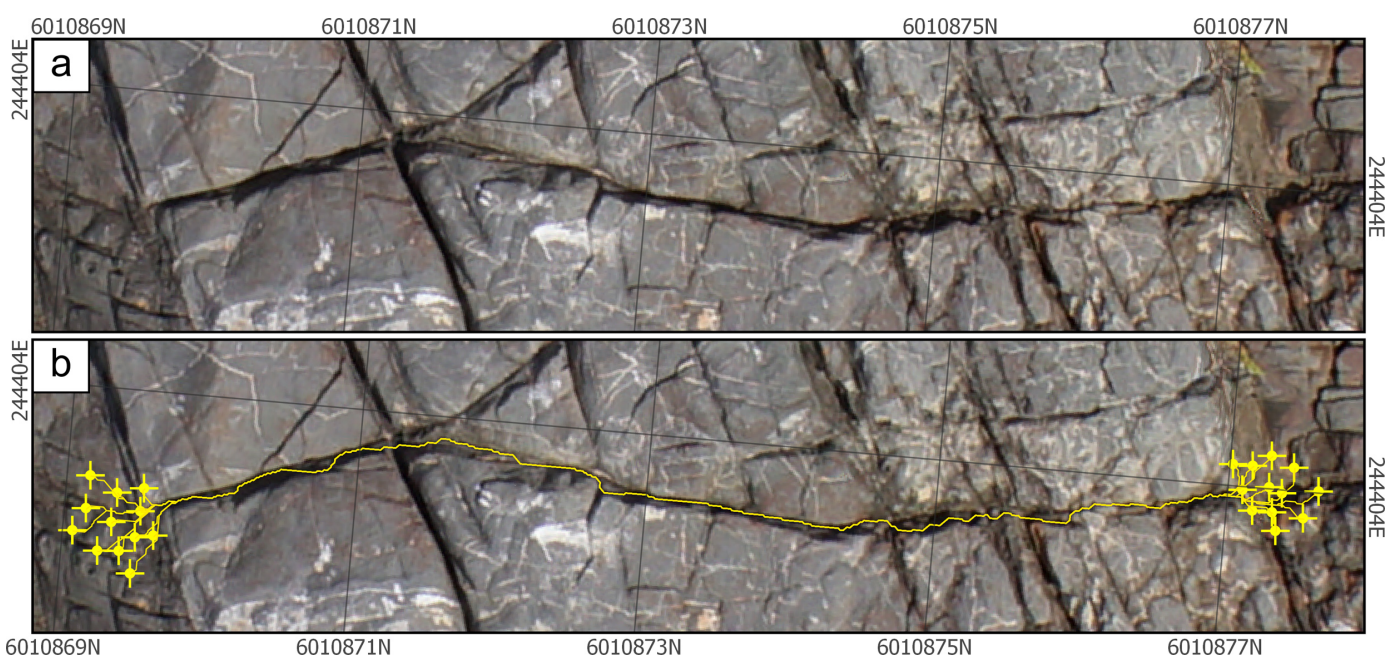

Figure 7. Fracture (a) from the Bingie Bingie Point dataset showing that the majority of the resulting trace (b) consistently follows the same path despite variation in the location of the control points.

pass requires only local information such that the calculation time scales with trace length and not dataset size. This means the tool can be used to interpret arbitrarily large point clouds.

Finally, the computer-assisted philosophy behind our method keeps the expert in control of the entire digitisation process, allowing for data vetting and correction during digitisation. The approach ensures that the expert becomes familiar with the particular intricacies of each dataset, a key part of further data analysis and something that is not possible using fully automated methods but is essential for the creative process of understanding and interpreting spatial information.

\section{Conclusions}

We have described a least-cost-path-based method for the computer-assisted digitisation of structural traces in point cloud, image and raster datasets. The method enhances an expert's ability to extract geological information from the wide range of high-resolution data available to geoscientists while reducing the required time and effort. The advantages of the method can be summarised as follows:

- allows for expert-guided interpretation in a way that seamlessly utilises computing power to significantly optimise the interpretation process and improve objectivity and consistency;
- can be applied to both raster and point cloud datasets (this is particularly significant in situations for which complex topography prevents a more conventional 2.5D raster-based workflow);

- requires only local knowledge of a dataset so that the total dataset size does not affect performance, thereby allowing for the computer-assisted interpretation of exceedingly large datasets;

- and is implemented as two freely available and opensource plugins for the widely used CloudCompare and QGIS software packages.

Data availability. The datasets used for the Bingie Bingie Point and Cape Woolamai case studies are freely available from https://doi.org/10.4225/03/5981b31091af9 (Thiele et al., 2017). The bathymetric and vertical gravity gradient datasets (Sandwell et al., 2014) used for the oceanic fracture zone example can be downloaded from the University of California San Diego at http://topex. ucsd.edu/grav_outreach/, while the Greendale Fault lidar dataset is available on request from the authors of Duffy et al. (2012). 


\section{Appendix A}

We outline five simple cost functions that give reasonable results for different structure types. Each function is designed to give values between 0 and 1 , allowing combinations of functions to be used (by summation) and to work on both unstructured datasets (i.e. point clouds) and structured datasets (images). Hence, we do not present any cost functions that rely on commonly used image processing techniques such as edge enhancement, although these could be easily incorporated for raster datasets. These functions are implemented directly in the Compass plugin, while simple QGIS functionality can be used to apply them to raster data for use with GeoTrace.

\section{A1 Colour brightness}

The brightness of an edge's end colour $\left(e_{\mathrm{RGB}}\right)$ can be mapped directly to edge cost (the brightness of an edge's start colour will be incorporated into the previous edge in the path). Despite its simplicity, this function (Eq. A1) is surprisingly effective at picking fracture traces, which are typically darker than their surroundings due to shadowing. Similarly, bright traces such as thin quartz or calcite veins can be identified using the opposite of this cost function (Eq. A2). Note that the division by 3 ensures that the function maps to the $0-1$ range (assuming red, green and blue values also range from 0 to 1$)$.

$\operatorname{cost}=\frac{\boldsymbol{e}_{\mathrm{R}}+\boldsymbol{e}_{\mathrm{G}}+\boldsymbol{e}_{\mathrm{B}}}{3}$

$\operatorname{cost}=1-\frac{\boldsymbol{e}_{\mathrm{R}}+\boldsymbol{e}_{\mathrm{G}}+\boldsymbol{e}_{\mathrm{B}}}{3}$

\section{A2 Colour similarity}

A similar cost function, based on colour similarity rather than brightness alone, is useful in more generic situations in which traces have a distinctive colour but are not necessarily darker or lighter than their surroundings. This function (Eq. A3) considers an edge to be low cost if (1) the start and end colours are similar and (2) the start and end colours are similar to the colour of the start and end of the trace $\left(B_{\mathrm{RGB}}\right.$ and $\left.E_{\mathrm{RGB}}\right)$, minimising the along-path gradient and maximising similarity with the trace start and end points. This function works well when traces have a specific colour, such as for cemented joints, though it is comparatively slow compared to the brightness-based functions described above due to the large number of square roots. Similarly to the previous equations, the factors of $\sqrt{3}$ ensure that the function maps to the $0-1$ range.

$\operatorname{cost}=$

$\frac{1}{2}\left(\frac{\left|s_{\mathrm{RGB}}-\boldsymbol{e}_{\mathrm{RGB}}\right|}{\sqrt{3}}+\frac{\left|\boldsymbol{s}_{\mathrm{RGB}}-\boldsymbol{B}_{\mathrm{RGB}}\right|+\left|s_{\mathrm{RGB}}-\boldsymbol{E}_{\mathrm{RGB}}\right|+\left|\boldsymbol{e}_{\mathrm{RGB}}-\boldsymbol{B}_{\mathrm{RGB}}\right|+\left|\boldsymbol{e}_{\mathrm{RGB}}-\boldsymbol{E}_{\mathrm{RGB}}\right|}{4 \sqrt{3}}\right)$

\section{A3 Gradient}

The previous cost functions are useful for identifying discrete structural traces such as faults, joints or thin veins, but will not be sensitive to lithological contacts. Lithological contacts are typically defined by changes in colour, and hence we base a cost function around colour gradient to identify them. This function (Eq. A4) evaluates the gradient $G[N]$ of the magnitude of the colour vectors across the start and end neighbourhoods $N_{\text {start }}$ and $N_{\text {end }}$. To calculate the gradient for point cloud data, we use a simple method that calculates the average distance-weighted point-to-point gradient for each neighbourhood. More complex methods would highlight contacts better, but at a computational cost. For raster data, we implement a Sobel filter to achieve equivalent results.

An upper limit $(l)$ is applied to the gradient in order to maintain a cost value between 0 and 1 . A reasonable value for this limit can be approximated by dividing the maximum change in colour magnitude $(\sqrt{3})$ by the average distance between data points. This cost function can also be improved by $\log$ transforming it to increase the importance of gradients resulting from more subtle features.

$\operatorname{cost}=1-\frac{\min \left(G\left[\mathbf{N}_{\text {start }}\right]+G\left[\mathbf{N}_{\text {end }}\right], l\right)}{l}$

\section{A4 Curvature}

In some situations, resolution is high enough that structural traces, fractures in particular, appear as topographic ridges or valleys. Hence, we include a final cost function (Eq. A5) which considers points with a high curvature as low cost, allowing paths to "follow" ridges and valleys along which $C[N]$ calculates the mean curvature of a point or pixel neighbourhood $N$, and $l$ is an arbitrarily large upper limit (that allows the $\log$ curvature to scale from 0 to 1 ). Note that calculating the mean curvature of a neighbourhood is computationally expensive, so this cost function performs significantly slower than the previously described ones unless curvature is pre-computed.

$\operatorname{cost}=1-\frac{\min \left(\log \left(C\left[\mathbf{N}_{\mathrm{end}}\right]\right), l\right)}{l}$ 
Author contributions. ST and LG developed the methodology described in this study. ST implemented it in CloudCompare and LG implemented it in QGIS. All of the authors contributed to the case studies and helped prepare the paper.

Competing interests. The authors declare that they have no conflict of interest.

Acknowledgements. The authors would like to gratefully acknowledge Daniel Girardeau-Montaut and other CloudCompare developers for creating a fantastic software package and for their assistance creating the Compass plugin. Samuel T. Thiele was supported by a Westpac Future Leaders Scholarship and an Australian Postgraduate Award. Lachlan Grose was supported by an Australian Postgraduate Award. Anindita Samsu was supported by a Monash University Faculty of Science Dean's International Postgraduate Research Scholarship and an American Association of Petroleum Geologists Grants-in-Aid award. Finally, we acknowledge Andrea Bistacchi and Thomas Scheiber for their insightful and constructive reviews.

Edited by: Gwenn Peron-Pinvidic

Reviewed by: Andrea Bistacchi and Thomas Scheiber

\section{References}

Banerjee, S. and Mitra, S.: Fold-thrust styles in the Absaroka thrust sheet, Caribou National Forest area, Idaho-Wyoming thrust belt, J. Struct. Geol., 27, 51-65, https://doi.org/10.1016/j.jsg.2004.07.004, 2005.

Bemis, S. P., Micklethwaite, S., Turner, D., James, M. R., Akciz, S., Thiele, S. T., and Bangash, H. A.: Ground-based and UAVBased photogrammetry: A multi-scale, high-resolution mapping tool for structural geology and paleoseismology, J. Struct. Geol., 69, 163-178, https://doi.org/10.1016/j.jsg.2014.10.007, 2014.

Blaikie, T. N., Betts, P. G., Armit, R. J., and Ailleres, L.: The ca. 1740-1710 Ma Leichhardt Event: Inversion of a continental rift and revision of the tectonic evolution of the North Australian Craton, Precambrian Res., 292, 75-92, https://doi.org/10.1016/j.precamres.2017.02.003, 2017.

Bond, C. E., Gibbs, A. D., Shipton, Z. K., and Jones, S.: What do you think this is? "Conceptual uncertainty" in geoscience interpretation, GSA today, 17, 4-10, https://doi.org/10.1130/gsat01711a.1, 2007.

Buckley, M. and Yang, J.: Regularised shortest-path extraction, Pattern Recogn. Lett., 18, 621-629, https://doi.org/10.1016/s01678655(97)00076-7, 1997.

Buckley, S. J., Howell, J. A., Enge, H. D., and Kurz, T. H.: Terrestrial laser scanning in geology: data acquisition, processing and accuracy considerations, J. Geol. Soc., 165, 625-638, https://doi.org/10.1144/0016-76492007-100, 2008.

Cruden, A., Vollgger, S., Dering, G., and Micklethwaite, S.: High Spatial Resolution Mapping of Dykes Using Unmanned Aerial Vehicle (UAV) Photogrammetry: New Insights On Emplacement Processes, Acta Geol. Sin.-Engl., 90, 52-53, https://doi.org/10.1111/1755-6724.12883, 2016.
Dering, G., Micklethwaite, S., Barnes, S. J., Fiorentini, M., Cruden, A., and Tohver, E.: An Elevated Perspective: Dyke-Related Fracture Networks Analysed with Uav Photogrammetry, Acta Geol. Sin.-Engl., 90, 54-55, https://doi.org/10.1111/1755-6724.12884, 2016.

Dewez, T. J. B., Girardeau-Montaut, D., Allanic, C., and Rohmer, J.: FACETS : A CLOUDCOMPARE PLUGIN TO EXTRACT GEOLOGICAL PLANES FROM UNSTRUCTURED 3D POINT CLOUDS, Int. Arch. Photogramm. Remote Sens. Spatial Inf. Sci., XLI-B5, 799-804, https://doi.org/10.5194/isprs-archivesXLI-B5-799-2016, 2016.

Dijkstra, E. W.: A note on two problems in connexion with graphs, Numer. Math., 1, 269-271, https://doi.org/10.1007/bf01386390, 1959.

Duffy, B., Quigley, M., Barrell, D. J. A., Van Dissen, R., Stahl, T., Leprince, S., McInnes, C., and Bilderback, E.: Fault kinematics and surface deformation across a releasing bend during the 2010 MW 7.1 Darfield, New Zealand, earthquake revealed by differential LiDAR and cadastral surveying, Geol. Soc. Am. Bull., 125, 420-431, https://doi.org/10.1130/b30753.1, 2012.

García-Sellés, D., Falivene, O., Arbués, P., Gratacos, O., Tavani, S., and Muñoz, J. A.: Supervised identification and reconstruction of near-planar geological surfaces from terrestrial laser scanning, Comput. Geosci., 37, 1584-1594, https://doi.org/10.1016/j.cageo.2011.03.007, 2011.

Gray, D. R.: Tectonics of the southeastern Australian Lachlan Fold Belt: structural and thermal aspects, Geological Society, London, Special Publications, 121, 149-177, https://doi.org/10.1144/gsl.sp.1997.121.01.07, 1997.

Holden, E.-J., Wong, J. C., Kovesi, P., Wedge, D., Dentith, M., and Bagas, L.: Identifying structural complexity in aeromagnetic data: An image analysis approach to greenfields gold exploration, Ore Geol. Rev., 46, 47-59, 10.1016/j.oregeorev.2011.11.002, 2012.

Holden, E.-J., Wong, J. C., Wedge, D., Martis, M., Lindsay, M., and Gessner, K.: Improving assessment of geological structure interpretation of magnetic data: An advanced data analytics approach, Comput. Geosci., 87, 101-111, https://doi.org/10.1016/j.cageo.2015.11.010, 2016.

Jaboyedoff, M., Couture, R., and Locat, P.: Structural analysis of Turtle Mountain (Alberta) using digital elevation model: Toward a progressive failure, Geomorphology, 103, 5-16, https://doi.org/10.1016/j.geomorph.2008.04.012, 2009.

Jinfei, W. and Howarth, P. J.: Use of the Hough transform in automated lineament, IEEE T. Geosci. Remote, 28, 561-567, https://doi.org/10.1109/tgrs.1990.572949, 1990.

Jones, R. R., McCaffrey, K. J. W., Clegg, P., Wilson, R. W., Holliman, N. S., Holdsworth, R. E., Imber, J., and Waggott, S.: Integration of regional to outcrop digital data: $3 \mathrm{D}$ visualisation of multi-scale geological models, Comput. Geosci., 35, 4-18, https://doi.org/10.1016/j.cageo.2007.09.007, 2009.

Kovesi, P.: Image features from phase congruency, Videre, J. Comput. Vision Res., 1.3, 1-26, 1999.

Lato, M. J. and Vöge, M.: Automated mapping of rock discontinuities in $3 \mathrm{D}$ lidar and photogrammetry models, Int. J. Rock Mech. Min., 54, 150-158, https://doi.org/10.1016/j.ijrmms.2012.06.003, 2012.

Masoud, A. and Koike, K.: Applicability of computeraided comprehensive tool (LINDA: LINeament Detec- 
tion and Analysis) and shaded digital elevation model for characterizing and interpreting morphotectonic features from lineaments, Comput. Geosci., 106, 89-100, https://doi.org/10.1016/j.cageo.2017.06.006, 2017.

Matthews, K. J., Müller, R. D., Wessel, P., and Whittaker, J. M.: The tectonic fabric of the ocean basins, J. Geophys. Res., 116, B12109, https://doi.org/10.1029/2011jb008413, 2011.

McCaffrey, K. J. W., Jones, R. R., Holdsworth, R. E., Wilson, R. W., Clegg, P., Imber, J., Holliman, N., and Trinks, I: Unlocking the spatial dimension: digital technologies and the future of geoscience fieldwork, J. Geol. Soc., 162, 927-938, https://doi.org/10.1144/0016-764905-017, 2005.

Pavlis, T. L. and Mason, K. A.: The New World of 3D Geologic Mapping, GSA Today, 27, 4-10, https://doi.org/10.1130/gsatg313a.1, 2017.

Pringle, J. K., Howell, J. A., Hodgetts, D., Westerman, A. R., and Hodgson, D. M.: Virtual outcrop models of petroleum reservoir analogues: a review of the current state-of-the-art, First break, 24, 33-42, https://doi.org/10.3997/1365-2397.2006005, 2006.

Richards, J. R. and Singleton, O. P.: Palaeozoic Victoria, Australia: Igneous rocks, ages and their interpretation, J. Geol. Soc. Aust., 28, 395-421, https://doi.org/10.1080/00167618108729178, 1981.

Sandwell, D. T. and Smith, W. H. F.: Global marine gravity from retracked Geosat and ERS-1 altimetry: Ridge segmentation versus spreading rate, J. Geophys. Res., 114, B01411, https://doi.org/10.1029/2008jb006008, 2009.

Sandwell, D. T., Muller, R. D., Smith, W. H. F., Garcia, E., and Francis, R.: New global marine gravity model from CryoSat-2 and Jason-1 reveals buried tectonic structure, Science, 346, 6567, https://doi.org/10.1126/science.1258213, 2014.

Scheiber, T., Fredin, O., Viola, G., Jarna, A., Gasser, D., and Lapinska-Viola, R.: Manual extraction of bedrock lineaments from high-resolution LiDAR data: methodological bias and human perception, GFF, 137, 362-372, https://doi.org/10.1080/11035897.2015.1085434, 2015.

Seers, T. D. and Hodgetts, D.: Extraction of three-dimensional fracture trace maps from calibrated image sequences, Geosphere, 12, 1323-1340, 2016.

Seton, M., Mortimer, N., Williams, S., Quilty, P., Gans, P., Meffre, S., Micklethwaite, S., Zahirovic, S., Moore, J., and Matthews, K. J.: Melanesian back-arc basin and arc development: Constraints from the eastern Coral Sea, Gondwana Res., 39, 77-95, https://doi.org/10.1016/j.gr.2016.06.011, 2016.

Smith, M. W., Carrivick, J. L., and Quincey, D. J.: Structure from motion photogrammetry in physical geography, Prog. Phys. Geog., 40, 247-275, https://doi.org/10.1177/0309133315615805, 2016.

Sobel, I.: An isotropic $3 \times 3$ image gradient operator, Machine vision for three-dimensional scenes, 376-379, https://doi.org/10.13140/RG.2.1.1912.4965, 1990.
Sun, C. and Pallottino, S.: Circular shortest path in images, Pattern Recogn., 36, 709-719, https://doi.org/10.1016/s00313203(02)00085-7, 2003.

Thiele, S. T., Micklethwaite, S., Bourke, P., Verrall, M., and Kovesi, P.: Insights into the mechanics of en-échelon sigmoidal vein formation using ultra-high resolution photogrammetry and computed tomography, J. Struct. Geol., 77, 27-44, https://doi.org/10.1016/j.jsg.2015.05.006, 2015.

Thiele, S. T., Vollgger S., Samsu, A.: GeoTrace and Compass rapid trace-mapping (example data), https://doi.org/10.4225/03/5981b31091af9, 2017.

Tzong-Dar, W. and Lee, M. T.: Geological lineament and shoreline detection in SAR images, Int. Geosci. Remote. Se., Barcelona, Spain, Spain, 23-28 July 2007.

van der Walt, S., Colbert, S. C., and Varoquaux, G.: The NumPy Array: A Structure for Efficient Numerical Computation, Comput. Sci. Eng., 13, 22-30, https://doi.org/10.1109/MCSE.2011.37, 2011.

van der Walt, S., Schönberger, J. L., Nunez-Iglesias, J., Boulogne, F., Warner, J. D., Yager, N., Gouillart, E., and Yu, T.: scikit-image: image processing in Python, PeerJ, 2, e453, https://doi.org/10.7717/peerj.453, 2014.

Vasuki, Y., Holden, E.-J., Kovesi, P., and Micklethwaite, S.: Semiautomatic mapping of geological Structures using UAV-based photogrammetric data: An image analysis approach, Comput Geosci., 69, 22-32, https://doi.org/10.1016/j.cageo.2014.04.012, 2014.

Vincent, L.: Minimal path algorithms for the robust detection of linear features in gray images, Comp. Imag. Vis., 12, 331-338, 1998.

Vollgger, S. A. and Cruden, A. R.: Mapping folds and fractures in basement and cover rocks using UAV photogrammetry, Cape Liptrap and Cape Paterson, Victoria, Australia, J. Struct. Geol., 85, 168-187, https://doi.org/10.1016/j.jsg.2016.02.012, 2016.

Weatherall, P., Marks, K. M., Jakobsson, M., Schmitt, T., Tani, S., Arndt, J. E., Rovere, M., Chayes, D., Ferrini, V., and Wigley, R.: A new digital bathymetric model of the world's oceans, Earth Space Sci., 2, 331-345, https://doi.org/10.1002/2015ea000107, 2015.

Wessel, P., Matthews, K. J., Müller, R. D., Mazzoni, A., Whittaker, J. M., Myhill, R., and Chandler, M. T.: Semiautomatic fracture zone tracking, Geochem. Geophy. Geosy., 16, 2462-2472, https://doi.org/10.1002/2015gc005853, 2015.

Williams, S. E., Flament, N., and Müller, R. D.: Alignment between seafloor spreading directions and absolute plate motions through time, Geophys. Res. Lett., 43, 1472-1480, https://doi.org/10.1002/2015g1067155, 2016. 